\title{
TIBIAL COMPONENT IN REVISION OF TOTAL KNEE ARTHROPLASTY: COMPARISON BETWEEN CEMENTED AND HYBRID FIXATION
}

Francisco Fontes Cintra' ${ }^{1}$, Anthony Kerbes Yepéz', Marcos Gilbert Sucena Rasga' ${ }^{1}$, Marcelo Abagge ${ }^{2}$, Paulo Gilberto Cimbalista Alencar ${ }^{3}$

\section{ABSTRACT}

Objective: To compare the clinical, radiographic and medium-term follow-up results from two fixation methods for the tibial component in revision procedures on total knee prostheses: cemented (tray and stem) and hybrid (cemented tray and uncemented, nonporous canal-filling stem). Methods: Between August 1999 and November 2005, 30 revision procedures on total knee arthroplasties were performed on 26 patients, who were divided between group I (cemented fixation; 21 knees) and group II (hybrid fixation; nine knees). The mean follow-up was 52 months and no patients were lost from the follow up. Results: No differences in the scores from

\section{INTRODUCTION}

Total knee arthroplasty (TKA) is one of the best surgical procedures for treating osteoarthrosis and is becoming increasingly common in orthopedic practice. With increasing life expectancy and absolute numbers of this surgical procedure, it can be expected that the number of revision procedures performed will also increase. It has been forecast that in the United States, the number of TKA revision procedures will increase by around $600 \%$ by the year $2030^{(1)}$.

Most knees with TKA loosening present a metaphyseal bone defect that does not allow adequate fixation of implants designed for primary the WOMAC and Knee Society questionnaires were observed between the two groups. One patient in group I presented radiographic signs of loosening. Two patients (one in each group) complained of pain in the diaphyseal region, compatible with the location of the stem tip. The pedestal radiographic sign was observed in $89 \%$ of the knees with uncemented stems and in none of the cemented group. Conclusion: The comparative analysis between the two methods did not show any differences regarding clinical and radiographic parameters, or arthroplasty survival.

Keywords - Knee/surgery; Arthroplasty; Revision; Cementation arthroplasty $^{(2-4)}$. To resolve this issue, intramedullary nails can be used: these increase the stability of the fixation and diminish both the stress at the bone-cement interface and the rate of prosthesis loosening ${ }^{(4-11)}$. Moreover, they protect bone grafts by increasing the consolidation rate and reducing the incidence of graft fractures ${ }^{(2-4)}$. Most revision systems provide interchangeable nails of different diameters and the lengths, which can be used with or without cement. However, currently, there are divergences in the literature regarding the best fixation method ${ }^{(2,4-14)}$.

Different studies on the biomechanics of cemented fixation of revision prostheses have demonstrated marked improvements in the stability of bone-

\footnotetext{
1 - Orthopedist and Member of the Brazilian Society of Orthopedics and Traumatology (SBOT), São Paulo, SP, Brazil.

2 - PhD from the Federal University of Paraná (UFPR), Curitiba, PR, Brazil.

3 - PhD. Head of the Hip and Knee Surgery Group, Federal University of Paraná (UFPR), Curitiba, PR, Brazil.

Work performed at Hospital de Clínicas, Federal University of Paraná, Curitiba, PR.

Correspondence: R. Mutamba 62, Alphaville Campinas, 13098-357 Campinas, SP. E-mail: franciscocintra@hotmail.com

Work received for publication: November 2, 2010; accepted for publication: March 21, 2011.
}

The authors declare that there was no conflict of interest in conducting this work 
prosthesis fixation. Furthermore, the cement can be a carrier for antibiotics, which is useful in treating prosthetized knees presenting previous infection. However, with this type of fixation, a higher rate of protection against mechanical stress, poor nail alignment and technical difficulty in removing cement from the canal, if a new revision becomes necessary, have been observed ${ }^{(2-4,10,12-15)}$.

The fixation is said to be hybrid when components involving thick uncemented nails that fill the canal are used, and the tray is fixed to the metaphyseal bone with cement. In vitro studies have shown that when this method is used, the same rate of bone-prosthesis stability is achieved, comparing long uncemented nails that fill the canal with short cemented nails. In addition, the position of the implant in the intramedullary canal is more accurate. If a new revision of the arthroplasty becomes necessary, the surgical procedure will be easier, given that no cement needs to be removed from the medullary canal. Nonetheless, some studies have demonstrated that the prosthesis has a shorter survival rate, compared with cemented fixation, and greater incidence of diaphyseal pain $^{(2,4,5-9,11,12-14,16)}$.

The frequency of loosening of the tibial component is greater than that of the femoral component, mainly because of the length of the support from the tibial tray ${ }^{(13)}$. Several authors have published results using different fixation methods, but few studies have compared techniques. The aim of the present study was to compare the clinical, radiographic and medium-term followup results from two methods for fixation of the tibial component in surgical revision procedures on total knee prostheses: cemented (tray and nail) and hybrid (cemented tray and uncemented nonporous nail that fills the canal).

\section{MATERIAL AND METHODS}

Between August 1999 and November 2005, 53 consecutive surgical revision procedures on knee arthroplasty were performed at our hospital. The patients with cemented fixation (cemented tibial tray and nail) and hybrid (cemented tray and uncemented non-porous nail that fills the canal), with a minimum of 24 months of postoperative follow up, were included in this study. We excluded 16 patients who did not have these two types of implants. Thus, 37 revisions fulfilled these criteria, and their results were assessed retrospectively. There were no losses from the follow-up. A further seven patients (six knees) were withdrawn from the study: three because of acute postoperative periprosthetic infection (one with cemented fixation and two with hybrid fixation), three who died for reasons unrelated to the arthroplasty (and for whom, therefore, some postoperative data was missing) and one who underwent another revision procedure 16 months later (in which revision of the femoral component alone was performed; the tibial component with an uncemented nail was fixed and was not replaced). The final group included 30 revisions in 26 patients. Three of these patients died ( 24 months after the surgery), but because their radiographic and clinical examination data were complete, these three patients were included.

There were 14 female patients and 12 male patients. Their mean age at the time of the surgery was 62.8 years (ranging from 23 to 82 years). We followed up the patients for an average of 53 months (ranging from 27 to 96 months). Fourteen revisions were performed on the right side and sixteen on the left side.

Twenty-three knee arthroplasty revisions were performed because of aseptic loosening (of which three were associated with periprosthetic tibial fractures) and seven were due to periprosthetic infection. The prostheses with aseptic loosening were revised with cemented fixation in the cases of 15 knees and with a hybrid in eight. The seven infected prostheses were revised in two stages, and cemented fixation was used in five knees and hybrid in two. None of the patients had presented reinfection by the time when this study was concluded.

The patients were divided into two groups: group I - cemented revision (21 knees in 17 patients); and group II - hybrid revision (nine knees in nine patients). The following data were analyzed on the preoperative radiographs:

a) Position of the implants;

b) Signs of fractures;

c) Bone loss: the defects were categorized according to the classification system of the Anderson Orthopedic 
Research Institute (AORI), as proposed by Engh(3) (Figures 1A, B and C).

In the postoperative radiographs (immediate and late postoperative periods), we analyzed the following parameters $^{(4,5,7,10)}$ :

d) Position of the nail in the medullary canal;

e) Presence of the "pedestal" sign and the cortical reaction (Figure 2);

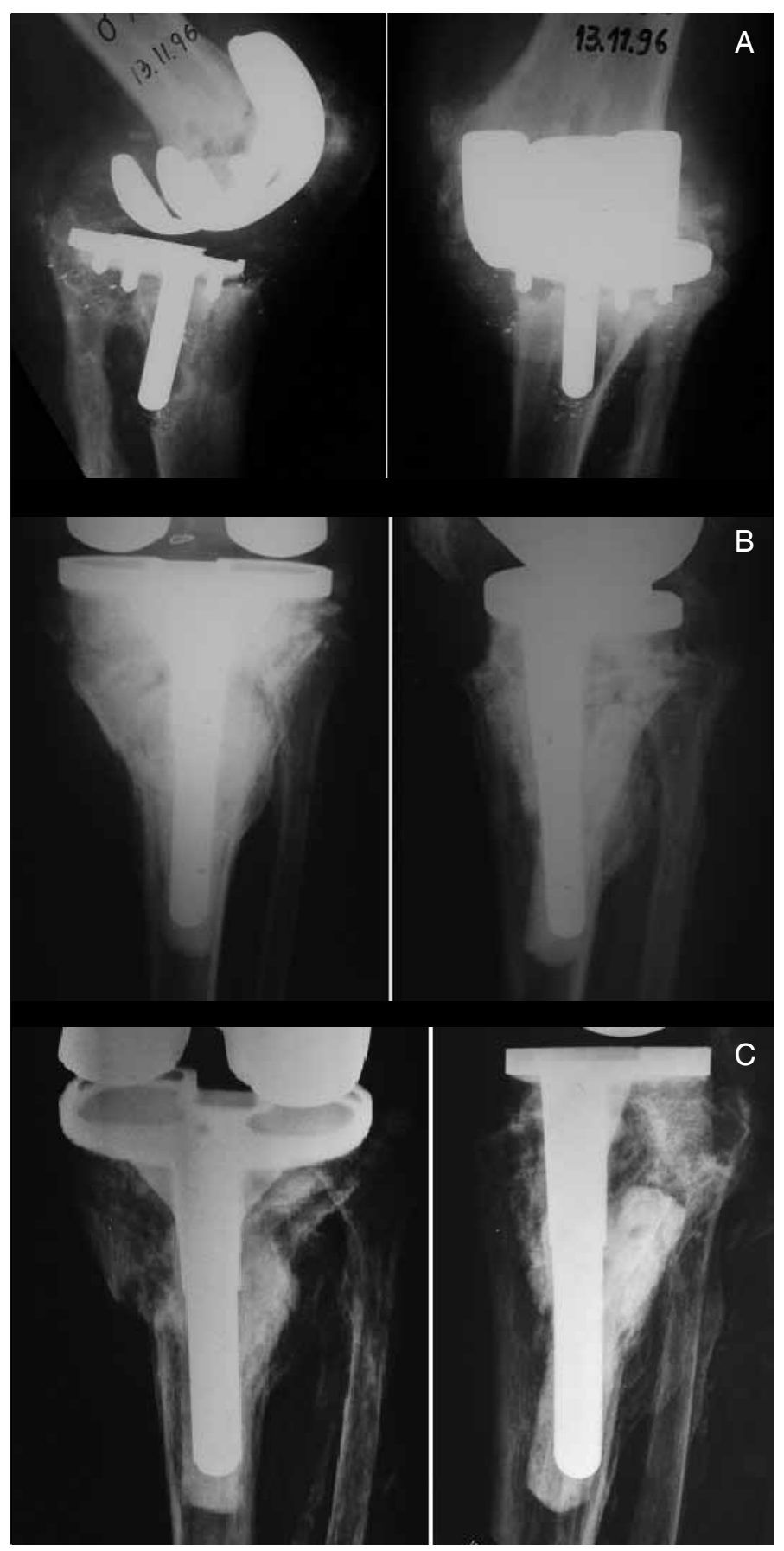

Figure 1 - (A) Loosening of arthroplasty with AORI 3 tibial bone loss. (B) Immediate postoperative period after treatment with structural graft and cemented nail. (C) 70 months after the operation: the prosthesis remains stable and without radiolucent lines. f) Percentage filling of the canal in hybrid fixation: the measurement was made in the AP and lateral radiographic views. In the region of the last $2 \mathrm{~cm}$ of the nail tip, we measured the diameter of the medullary canal and nail. We then calculated the percentage of the medullary canal that the nail was occupying. This proportion ought to be at least $70 \%{ }^{(12)}$;

g) Signs of fixation failure: migration of the implant on serial radiographs and a complete radiolucent line around the implant (Figure 3).

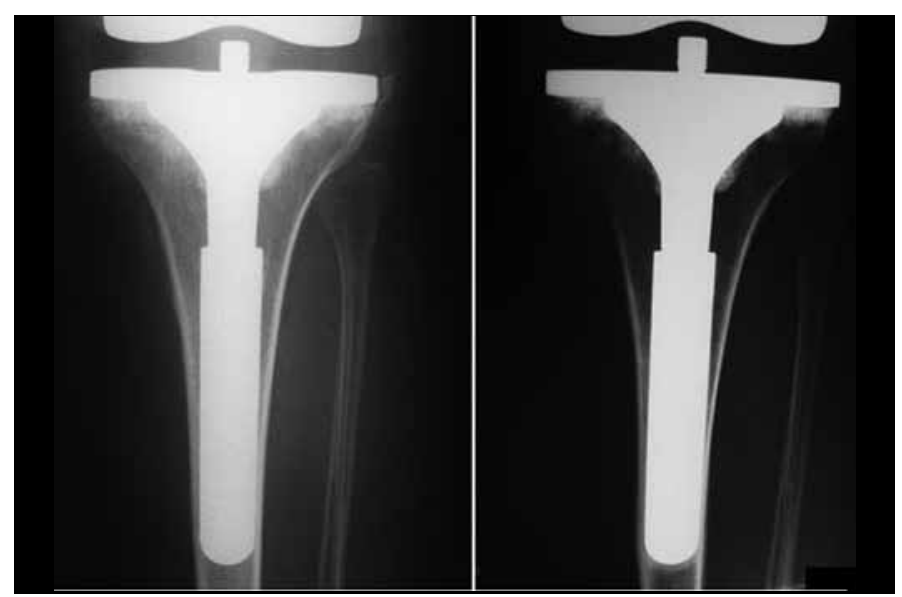

Figure 2 - Evolution of "pedestal" sign: immediate postoperative period (left) and 40 months after the operation (right).

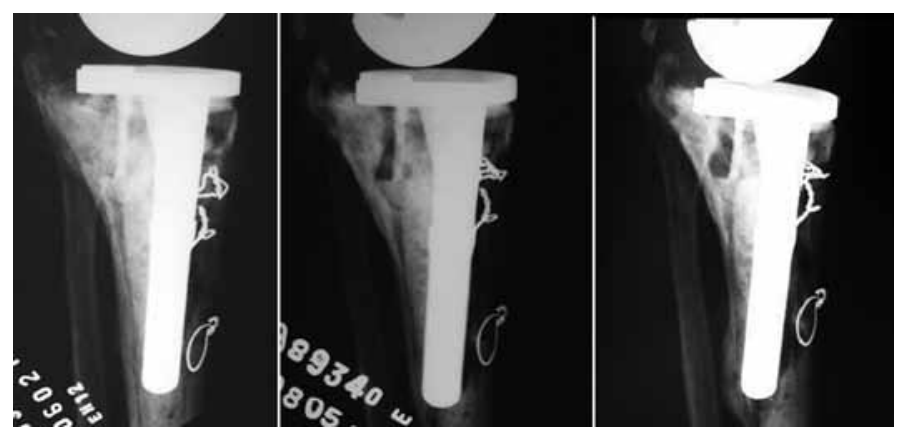

Figure 3 - Radiographic evolution of prosthesis loosening. Note the posterior cortical reaction and nail migration.

We used implants with posterior stabilization in all cases, and there was no need to increase the constriction level.

For the cemented revisions $\left(\mathrm{PFC}\right.$, Johnson ${ }^{\circledR}$ ), we used nails of sufficient length to fix the prosthesis in good-quality bone (while trying to use one that was as short as possible). We cemented the canal manually. We used a plastic blocker at around $1 \mathrm{~cm}$ from the nail tip in 14 of the 21 surgeries. In the other cases, we used a bone blocker. All the revisions were performed using cement that contained antibiotic 
premixed by the manufacturer (several brands; 1 g of gentamicin per dose).

For the hybrid revisions (Search, Aesculap ${ }^{\circledR}$ ), we used manual milling bits of progressive diameter until reaching reasonable mechanical resistance (resistance to rotating the milling bit when using one hand), thereby avoiding excessive milling (the milling bit should not penetrate beyond the endosteum). The cement was implanted below the tibial tray, and a nonporous nail of the same diameter as the last milling bit was introduced in a position central to the medullary canal. Since eccentric nails were unavailable, we used a tibial tray of smaller diameter in two knees, given that these knees presented eccentric medullary canals. This procedure had the aim of avoiding abrasion between the implant and soft tissues after the surgery.

The indication for the implant to be used depended on the type of prosthesis available at the time of the surgery, according to the tendering process used by the hospital among qualified companies.

To analyze the clinical results, we used the Womac questionnaire and the Knee Society Score (KSS) on 17 patients (nine from group I and eight from group II). We excluded 13 patients from this part of the study (12 in group I and one in group II) because of the clinical comorbidities that they presented ${ }^{(4,5,7,10,11)}$ : seven who presented systemic conditions (three with spinal stenosis, two with juvenile rheumatoid arthritis, one with stroke sequelae and one with Alzheimer's disease); the three who died (causes unrelated to arthroplasty); the two who evolved with postoperative periprosthetic femoral fractures; and one who underwent a new revision 36 months later, due to failure of the femoral component of the arthroplasty.

We asked the patients who reported feeling pain in the diaphyseal region of the tibia to mark out the pain location using a pen, in order to evaluate whether there was any radiographic correlation with the symptoms ${ }^{(4,16)}$.

The clinical results were analyzed using the MannWhitney statistical method.

\section{RESULTS}

Five knees presented AORI 1 bone defects, which were filled with ground-up homologous bone. Metal wedges and blocks were used in seven knees with
AORI 2. Eight knees presented extensive AORI 3 defects, and these were treated with structural homologous grafts (six with cemented fixation and two with hybrid). Six of these defects became completely consolidated after one year; one of them only achieved partial consolidation (the posterior part of the graft showed failures on serial radiographs); and the last of them did not unite, although the arthroplasty remained stable, functional and without signs of loosening or migration on serial radiographs.

Two of the 21 nails in group I (cemented) were introduced into the canal in an eccentric position. All the nails in group II (hybrid) were in a central position.

Eight of the nine nails $(89 \%)$ in group II, but none in group I, developed the "pedestal" sign on postoperative serial radiographs (Figure 2). One patient in group I presented diaphyseal cortical hypertrophy in the region of the nail tip, five years after the surgery.

The mean percentage filling of the canal in group II was $77 \%$ (range: 64 to $95 \%$ ).

Two failures occurred, both in group I. The first patient was the one who presented cortical hypertrophy: the tibial implant evolved with distal migration and loosening of the arthroplasty, seven years after the surgery (Figure 3). This patient presented major preoperative bone loss (AORI 3), which was treated with a structural graft. We performed osteotomy on the anterior tuberosity of the tibia, for better exposure, which contributed towards the poor cementation of the nail (the cement leaked out through the osteotomy, during pressurization, such that the cement layer formed irregularly and with bubbles). Both of these events probably contributed towards the failure. This patient was scheduled for a new revision. The second patient presented a fracture in the femoral structural graft, 36 months after the surgery, and this was revised using a non-conventional endoprosthesis. The tibial implant was shown to be fixed, both clinically and radiographically. Although revision was indicated in this case, there were no clinical or radiological signs of loosening of the tibial prosthesis.

The mean KSS result for the knees in group I was 89.6 (range: 60-98) and for the knees in group II, 90 (range: 82-100). There was no statistical difference in these results $(p=0.758)$. The mean result from the 
Womac questionnaire was 82 (range: 64-98) for the knees in group I and 86 (range: 72-98) for the knees in group II, also without any statistical difference $(p=0.758)$. Although the mean length of the postoperative evaluation was greater in group I (62 months versus 42 months), there was no statistical difference between these follow up periods $(p=0.071)$.

For the two patients who complained of diaphyseal pain, there was a correlation between its location (marked out on the patient's skin) and the position of the nail tip on the radiograph. There were no specific radiographic signs that could be correlated with the pain.

The three patients who died presented radiographically fixed arthroplasties. Before they died, they made few complaints of pain and had good walking capacity. Their range of motion was good (full active extension, and active flexion of at least $100^{\circ}$ ), and their knees presented competent ligaments. We did not apply the questionnaires to these patients because they did not form part of the clinic's protocol before their deaths.

\section{DISCUSSION}

Revision surgery on knee arthroplasty presents a range of challenges that can be overcome through correctly choosing the implant ${ }^{(2-19)}$. Orthopedists need to identify the causes of failure of the arthroplasty previously used, in order to avoid poor results ${ }^{(2)}$. Stable and long-lasting fixation of the arthroplasty components to the bone is an integral part of the surgery, but the best method has not yet been defined $^{(2-14)}$.

The knee for which arthroplasty revision will be indicated usually presents poor bone quality (due to osteolysis and protection against mechanical stress) and/or bone losses (caused by a fracture, for example). Use of intramedullary nails diminishes the transmission of load to the metaphysis by 30 to $40 \%$ and increases the implant-bone contact area. These two factors prevent early loosening of the prosthesis $^{(2,3)}$.

Biomechanical tests have been conducted by different authors in order to clarify which fixation method is best. Comparison between the results from axial and eccentric loading on the tibias of cadavers showed that there was no difference in relation to micromovement or migration, either when using cemented fixation or when using hybrid fixation with long nails. It was concluded that both systems could provide stability and adequate fixation ${ }^{(12-14)}$. In the case of AORI 3 defects that were treated using structural grafts, implantation of cemented nails seemed to be a prudent choice ${ }^{(14)}$.

In our study, the positioning of the nails was better with hybrid fixation, given that all of them were in a central position, compared with cemented fixation. Nonetheless, no implant loosening or diaphyseal pain was observed. Since we did not use eccentric nails for hybrid fixation, the two knees that were revised using a tibial tray of smaller size did not present any impairment of the result or of fixation ${ }^{(2,4,9-11,18,19)}$.

Since hybrid fixation nails do not present microporosity (and therefore do not promote secondary stabilization through bone growth), they only add mechanical stability. It is known that, a few months after surgery, the bone around the nails may present the phenomenon of bone relaxation, which may lead to micromovements of the implant. The cementbone interface below the tibial tray should therefore provide all of the long-lasting implant-bone fixation for surgical success. This variable has been indicated by some authors to be the main cause of failure of this type of fixation, given that the metaphyseal bone generally is of poor quality. The tips of thick nails may cause pain in some patients, but this was a rare event in our study, affecting only two patients, one in

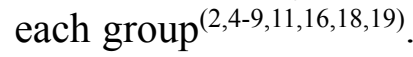

Few studies on the results from implants with medullary nails for knee arthroplasty revision have been published, and most of them were retrospective. After 14 years of follow-up, the Finnish arthroplasty register concluded that cemented fixation presented better survival than shown by hybrid uncemented fixation. However, there was no mention of the caliber of the hybrid implant. Many prostheses used in the past had thin nails, and in vivo and in vitro trials have already shown that these present unsatisfactory results $^{(14)}$. In a comparative study, Fehring et al ${ }^{(11)}$ showed that cemented nails presented better results, but the comparison was with short uncemented nails with metaphyseal fixation. The percentage filling of the canal was not mentioned. Chon et $a l^{(9)}$ conducted a 
comparative study between cemented fixation versus hybrid, using long uncemented nails, and did not find any difference in survival between the implants after a mean follow-up of 44 months. Gofton et $a l^{(5)}$, Shannon et $a l^{(6)}$ and Bottner et $a l^{(8)}$ studied prosthesis survival with hybrid fixation (with long nails) and found that it ranged from $84 \%$ to $93 \%$ in mediumterm follow-up. We achieved 100\% survival among our cases of hybrid fixation with a mean of 42 months of follow up.

There is still no consensus regarding the "pedestal" radiographic sign at the tip of uncemented nails (Figure 2). The results from Fehring et al ${ }^{(11)}$ were the same as in our study (90\%). It seems that there is no clinical-radiographic correlation or association with periprosthetic loosening. Shannon and Trousdale put forward the hypothesis that there could be a reaction between bone and chromium-cobalt nails, which have a low module of elasticity. However, there have not been any studies on associations between such findings and future complications ${ }^{(6,11)}$.

\section{CONCLUSION}

The medium-term results from the comparative analysis between hybrid and cemented revisions of the tibial component did not demonstrate any differences in clinical, radiographic or survival parameters of the arthroplasties. In view of these similar results and the fact that cement removal from the medullary canal in cases of new revision is more difficult in cases of cemented nails, our current choice is to use hybrid revision.

\section{REFERENCES}

1. Kurtz S, Ong K, Lau E, Mowat F, Halpern M. Projections of primary and revision hip and knee arthroplasty in the United States from 2005 to 2030. J Bone Joint Surg Am. 2007;89(4):780-5.

2. Nelson CL, Lonner JH, Rand JA, Lotke PA. Strategies of stem fixation and the role of supplemental bone graft in revision total knee arthroplasty. J Bone Joint Surg Am. 2003;85(Suppl 1):S52-7.

3. Mabry TM, Hanssen AD. The role of stems and augments for bone loss in revision knee arthroplasty. J Arthroplasty. 2007;22(4 Suppl 1):56-60.

4. Barrack RL, Rorabeck C, Burt M, Sawhney J. Pain at the end of the stem after revision total knee arthroplasty. Clin Orthop Relat Res. 1999;(367):216-25.

5. Gofton WT, Tsigaras H, Butler RA, Patterson JJ, Barrack RL, Rorabeck CH. Revision total knee arthroplasty: fixation with modular stems. Clin Orthop Relat Res. 2002;(404):158-68.

6. Shannon BD, Klassen JF, Rand JA, Berry DJ, Trousdale RT. Revision total knee arthroplasty with cemented components and uncemented intramedullary stems. J Arthroplasty. 2003;18(7 Suppl 1):27-32.

7. Whiteside LA. Cementless fixation in revision total knee arthroplasty. Clin Orthop Relat Res. 2006;446:140-8.

8. Bottner F, Laskin R, Windsor RE, Haas SB. Hybrid component fixation in revision total knee arthroplasty. Clin Orthop Relat Res. 2006;446:127-31.

9. Chon JG, Lombardi AV Jr, Berend KR. Hybrid stem fixation in revision total knee arthroplasty (TKA). Surg Technol Int. 2004;12:214-20.

10. Whaley AL, Trousdale RT, Rand JA, Hanssen AD. Cemented long-stem revision total knee arthroplasty. J Arthroplasty. 2003;18(5):592-9.
11. Fehring TK, Odum S, Olekson C, Griffin WL, Mason JB, McCoy TH. Stem fixation in revision total knee arthroplasty: a comparative analysis. Clin Orthop Relat Res. 2003;(416):217-24.

12. Jazrawi LM, Bai B, Kummer FJ, Hiebert R, Stuchin SA. The effect of stem modularity and mode of fixation on tibial component stability in revision total knee arthroplasty. J Arthroplasty. 2001;16(6):759-67.

13. Conditt MA, Parsley BS, Alexander JW, Doherty SD, Noble PC. The optimal strategy for stable tibial fixation in revision total knee arthroplasty. J Arthroplasty. 2004;19(7 Suppl 2):113-8.

14. Completo A, Simões JA, Fonseca F, Oliveira M. The influence of different tibial stem designs in load sharing and stability at the cement-bone interface in revision TKA. Knee. 2008;15(3):227-32.

15. Sheng PY, Konttinen L, Lehto M, Ogino D, Jämsen E, Nevalainen J, et al. Revision total knee arthroplasty: 1990 through 2002. A review of the Finnish arthroplasty registry. J Bone Joint Surg Am. 2006;88(7):1425-30.

16. Barrack RL, Stanley T, Burt M, Hopkins S. The effect of stem design on endof-stem pain in revision total knee arthroplasty. J Arthroplasty. 2004;19(7 Suppl 2):119-24.

17. Miller TT. Imaging of knee arthroplasty. Eur J Radiol. 2005;54(2):164-77.

18. Parsley BS, Sugano N, Bertolusso R, Conditt MA. Mechanical alignment of tibial stems in revision total knee arthroplasty. J Arthroplasty. 2003;18(7 Suppl 1):33-6.

19. Mahoney OM, Kinsey TL. Modular femoral offset stems facilitate joint line restoration in revision knee arthroplasty. Clin Orthop Relat Res. 2006;446:93-8. 